\title{
Chronic Psychological Stress and the Regulation of Pro-Inflammatory Cytokines: A Glucocorticoid-Resistance Model
}

Gregory E. Miller
Washington University

\author{
Sheldon Cohen \\ Carnegie Mellon University
}

\author{
A. Kim Ritchey \\ Children's Hospital of Pittsburgh
}

\begin{abstract}
This study examined whether chronic stress impairs the immune system's capacity to respond to hormonal signals that terminate inflammation. Fifty healthy adults were studied; half were parents of cancer patients, and half were parents of healthy children. Parents of cancer patients reported more psychological distress than parents of healthy children. They also had flatter diurnal slopes of cortisol secretion, primarily because of reduced output during the morning hours. There was also evidence that chronic stress impaired the immune system's response to anti-inflammatory signals: The capacity of a synthetic glucocorticoid hormone to suppress in vitro production of the pro-inflammatory cytokine interleukin- 6 was diminished among parents of cancer patients. Findings suggest a novel pathway by which chronic stress might alter the course of inflammatory disease.
\end{abstract}

Key words: psychoneuroimmunology, chronic stress, cortisol, pro-inflammatory cytokines, cancer, social support

Psychological stress has been linked with a broad array of adverse health outcomes. Studies have demonstrated that stress heightens risk for upper respiratory infection (Cohen et al., 1998; Cohen, Tyrrell, \& Smith, 1991; Sheridan \& Dobbs, 1994), accelerates the progression of coronary artery disease (Kaplan et al., 1983; Rozanski, Blumenthal, \& Kaplan, 1999), and exacerbates the course of autoimmune disorders (Grant, 1993; Rabin, 1999; Whitacre, Cummings, \& Griffin, 1995; Zautra, Burleson, Matt, \&

Gregory E. Miller, Department of Psychology, Washington University; Sheldon Cohen, Department of Psychology, Carnegie Mellon University; A. Kim Ritchey, Division of Hematology/Oncology, Children's Hospital of Pittsburgh, Pittsburgh, Pennsylvania.

This research was supported by grants from the National Heart, Lung, and Blood Institute (HL65111 and HL65112) to the Pittsburgh Mind-Body Center, the National Institute of Mental Health (MH50430, MH18269, and MH00721), and the American Heart Association (0160367Z).

We extend our gratitude to Edith Chen, Clemens Kirschbaum, Bruce McEwen, Bruce Rabin, and Nicolas Rohleder for their comments on earlier versions of this article. We also thank the Immunologic Monitoring and Diagnostic Laboratory at the University of Pittsburgh for performing the glucocorticoid sensitivity assays, Clemens Kirschbaum and his laboratory for performing the salivary cortisol assays, and Andrew Baum for his generous assistance throughout the study outside of the review process. Finally, we express our gratitude to the nursing and medical staff at the Children's Hospital of Pittsburgh, without whom this study would not have been possible.

Correspondence concerning this article should be addressed to Gregory E. Miller, Department of Psychology, Washington University, Campus Box 1125, One Brookings Drive, St. Louis, Missouri 63130. E-mail: gemiller@artsci.wustl.edu
Roth, 1994). To elucidate the mechanisms responsible for these effects, researchers have explored the relationship between psychological stress and the immune system, the body's chief defense against many diseases. Numerous links between stress and the immune response have been described. Studies have shown that stress is accompanied by suppressed lymphocyte proliferative responses, reduced control of latent herpes viruses, blunted humoral responses to immunization, and poorer wound healing (Cohen, Miller, \& Rabin, 2001; Herbert \& Cohen, 1993; Kiecolt-Glaser, Glaser, Gravenstein, Malarkey, \& Sheridan, 1996; Kiecolt-Glaser, Marucha, Malarkey, Mercado, \& Glaser, 1995; Marucha, KiecoltGlaser, \& Favagehi, 1998).

These findings have given rise to an immunosuppression model depicting relations between stress, immunity, and disease. The model's basic tenet is that stress heightens risk for adverse health outcomes by suppressing the immune response in a fashion that leaves the host vulnerable to opportunistic disease. Although the exact pathways responsible for this effect have not yet been elucidated, stress is assumed to downregulate immunity by (a) activating autonomic nervous system fibers that descend from the brain to lymphoid organs (Felten \& Felten, 1994), (b) triggering the secretion of hormones and neuropeptides that bind to white blood cells and alter their function (Blalock, 1994), and (c) inducing immunomodulatory coping behaviors, such as cigarette smoking and alcohol consumption (Kiecolt-Glaser \& Glaser, 1988). Under these conditions, the immune system's capacity to mount an effective response to challenge is diminished (Andersen, KiecoltGlaser, \& Glaser, 1994; Cohen \& Williamson, 1991).

The immunosuppression model makes important contributions to our understanding of stress-related disease. It is particularly 
good at explaining how stress might increase susceptibility to negative health outcomes that arise because of compromised host resistance. Infectious disease, some forms of cancer, and wound healing all fall into this category. An important limitation of the immunosuppression model, however, is that it does not offer a parsimonious explanation for how stress might influence diseases whose central feature is excessive inflammation. This is the case in many disease contexts: Inflammation plays a role in the pathogenesis of allergic, autoimmune, rheumatologic, and cardiovascular diseases and contributes to the formation of illness symptoms in many infectious diseases. These conditions seem to be exacerbated by stressful experience (Cohen et al., 1991, 1998; Grant, 1993; Rozanski et al., 1999; Whitacre et al., 1995; Wright, Rodriquez, \& Cohen, 1998; Zautra et al., 1994). However, it is difficult to see how the immunosuppression model could account for these findings. In fact, the most straightforward prediction that could be derived from it is that stress should improve disease course by suppressing the inflammatory response. The available data in humans generally do not bear out this prediction. ${ }^{1}$

So, how might the impact of stress on inflammatory conditions be explained? To answer this question, we propose a glucocorticoid-resistance model. Its basic premise is that chronic stress diminishes the immune system's sensitivity to glucocorticoid hormones that normally terminate the inflammatory cascade. The model begins with the notion that chronic stress elicits secretion of the hormonal products of the hypothalamic-pituitary-adrenocortical (HPA) and sympathetic adrenal medullary (SAM) axes. With continued exposure to high concentrations of these hormones, white blood cells mount a counterregulatory response and downregulate the expression and/or function of receptors responsible for binding glucocorticoid hormones. This receptor downregulation subsequently diminishes the immune system's capacity to respond to cortisol's anti-inflammatory actions. To the extent that this process occurs, inflammatory processes flourish and the course of disease subsequently worsens. ${ }^{2}$

This model's validity rests on a series of important assumptions. The first is that stressful circumstances can trigger ongoing secretion of hormones from the HPA and SAM axes. A number of studies have yielded support for this assumption, demonstrating that when environmental demands outstrip coping resources, the body's output of cortisol, epinephrine, and norepinephrine increases (Baum \& Grunberg, 1995; Kirschbaum \& Hellhammer, 1989; Weiner, 1992). These hormonal increases, however, are not uniformly observed. In patients with posttraumatic stress disorder, for instance, epinephrine and norepinephrine are persistently elevated, but cortisol secretion is persistently blunted (Yehuda, 2000). A second assumption of the model is that cortisol plays a central role in regulating the inflammatory response to infection and injury. Support for this assumption comes from studies demonstrating that disruption of the HPA axis predisposes rodents to chronic inflammatory illness, which resolves once hormonal pathways have been restored through the administration of synthetic glucocorticoids (Chrousos, 1995; Sternberg, Hill, et al., 1989; Sternberg, Young, et al., 1989). The model's final assumption is that with prolonged exposure to hormones, glucocorticoid receptors are downregulated and the immune system's sensitivity to the cortisol declines. Evidence of this phenomenon derives from clinical studies of inflammatory disease that show that in a subset of patients, long-term administration of synthetic glucocorticoid med- ications induces a glucocorticoid-resistance syndrome in which initially therapeutic dosages come to offer little in the way of relief (Chrousos, Detera-Wadleigh, \& Karl, 1993; DeRijk \& Sternberg, 1997; Leung, De Castro, Szefler, \& Chrousos, 1998). Although clinical evidence of this nature does not exist for SAM hormones, research suggests that epinephrine and norepinephrine can downregulate glucocorticoid receptor expression (DeRijk, Petrides, Deuster, Gold, \& Sternberg, 1996; Maccari et al., 1992).

In this article, we describe a study examining the basic principles of this model. It explores whether chronic psychological stress alters the capacity of a synthetic glucocorticoid (dexamethasone) to inhibit the in vitro production of the pro-inflammatory cytokines interleukin-1 $\beta$ (IL-1 $\beta$ ), interleukin-6 (IL-6), and tumor necrosis factor- $\alpha$ (TNF- $\alpha$ ). These cytokines are soluble molecules, released by white blood cells, that orchestrate inflammation. They have wide-ranging biological effects that include directing white blood cells toward sites of injury or infection, stimulating the production of other molecules involved in the inflammatory response, and enhancing the killing capacity of certain classes of white blood cells.

We accomplished this by studying a group of people experiencing a severe form of chronic psychological stress: having a child who is undergoing treatment for pediatric cancer. This situation represents a tremendous familial, economic, bureaucratic, and existential challenge for most parents. Indeed, a recent study that examined mothers of pediatric cancer patients found that approximately $20 \%$ had subclinical symptoms of posttraumatic stress disorder. More than 6\% met full Diagnostic and Statistical Manual of Mental Disorders (4th ed.; American Psychiatric Association, 1994) criteria for this disorder (Manne, Du Hamel, Gullelli, Sorgen, \& Redd, 1998). We expected that parents of cancer patients would show a decline in immune system glucocorticoid sensitivity compared with demographically matched parents of medically healthy children. This process would be manifest by a reduction in dexamethasone's capacity to suppress pro-inflammatory cytokine production.

We also explored affective, behavioral, and hormonal pathways that might be responsible for declines in immune system glucocorticoid sensitivity among parents of cancer patients. The major

\footnotetext{
${ }^{1}$ Although stress generally does not improve the course of inflammatory disease in humans, there is some evidence to suggest that symptoms of autoimmune disease may temporarily subside during extremely stressful circumstances (Nisipeanu \& Korczyn, 1993; Potter \& Zautra, 1997). Evidence of this phenomenon has also occasionally emerged in animal studies, both in rheumatoid arthritis (Rabin, 1999; Whitacre et al., 1995) and in infectious disease, where stress-related cortisol increases protected animals from the lethal consequences of respiratory inflammation (Sheridan et al. 1998).

${ }^{2}$ By using the term glucocorticoid resistance here, we do not mean to imply that stress induces a clinical syndrome where the immune system becomes completely unresponsive to glucocorticoids. We are simply arguing that stress will, to some extent, diminish the immune system's glucocorticoid sensitivity and, by doing so, will facilitate the continued expression of pro-inflammatory cytokines following infection and/or injury. In this sense, we are using the term in the same way that researchers in this field use immunosuppression-not to refer to a clinical entity but instead to a pattern of immune system changes that accompany chronic stress.
} 
affective pathway that we examined was depression, as it has been linked with diminished glucocorticoid sensitivity in both nervous system and immune system tissues (A. H. Miller, Pariante, \& Pearce, 1999; Gold, Goodwin, \& Chrousos, 1988a, 1988b). The behavioral pathways we explored were health practices that have been linked with the immune response in previous research (Cohen, Doyle, Skoner, Rabin, \& Gwaltney, 1997; Kiecolt-Glaser \& Glaser, 1988; G. E. Miller, Cohen, \& Herbert, 1999) and included smoking, alcohol consumption, physical activity, and sleep hygiene. To explore the contribution of hormonal pathways, we had parents collect saliva samples as they went about normal daily activities, and we used these samples to estimate diurnal patterns of cortisol secretion.

Social support can play a powerful role in buffering people from the biological consequences of stressful experience (Kamarck, Manuck, \& Jennings, 1990; Kirschbaum, Klauer, Sigrun-Heide, \& Hellhammer, 1995; Uchino, Cacioppo, \& Kiecolt-Glaser, 1996). Only a handful of studies, however, have examined whether this effect extends to the immune system (Baron, Cutrona, Hicklin, Russell, \& Lubaroff, 1990; Glaser et al., 1992; Glaser, KiecoltGlaser, Speicher, \& Holliday, 1985; Kiecolt-Glaser, Dura, Speicher, Trask, \& Glaser, 1991; Kiecolt-Glaser, Garner, et al., 1984; Kiecolt-Glaser, Speicher, Holliday, \& Glaser, 1984). To address this issue, we assessed the extent to which parents felt they had two forms of social support available to them: appraisal support, or the sense that one has others he or she can talk to about problems, and tangible support, or the feeling that one has others who will provide material aid when it is needed.

The primary hypotheses of this study were that (a) chronic stress would diminish the capacity of dexamethasone to suppress the production of pro-inflammatory cytokines; (b) negative affective states, maladaptive health practices, and disrupted cortisol secretion patterns would contribute to this process; and (c) high levels of social support would buffer parents from any reductions in glucocorticoid sensitivity that might arise from the stress of caring for a child with cancer.

\section{Method}

\section{Participants}

Fifty adults participated in this study. Half of them were parents of children undergoing active treatment for cancer; the remaining half were parents of medically healthy children. The two groups were matched with respect to age, gender, ethnicity, and marital status. All parents were in excellent health, defined as having (a) no history of chronic illness involving the cardiovascular, endocrine, or immune systems and (b) no use of prescription medications within the previous 3 months, with the exception of oral contraceptives. None of the female participants had been pregnant or lactating within 12 months of entering the study.

The parents of children with cancer were recruited from the Hematology/ Oncology Clinic at the Children's Hospital of Pittsburgh, Pittsburgh, Pennsylvania. To be eligible for the study, they had to be caring for a child undergoing active treatment for cancer who was 1-18 years old and living at home. Almost half of the parents were caring for a child with acute lymphoblastic leukemia (44\%), the most common form of childhood cancer. The remainder were caring for a child with neuroblastoma (16\%), hepatoblastoma (8\%), Wilms's tumor (4\%), osteogenic sarcoma (4\%), or other malignancies. The children had been diagnosed with cancer an average of 9.6 months prior to study entry $(S D=9.5$, range $=1-35$ months). In most cases, this diagnosis was for a primary tumor $(76 \%)$, although in some cases, it was for a recurrence (24\%). All children were undergoing chemotherapy at the time their parents entered the study. Additional treatment modalities included radiation therapy (60\%), surgery (40\%), and bone marrow transplantation (20\%). To estimate disease severity, we asked each child's attending physician to rate his or her prognosis on scale ranging from 1 (very poor) to 5 (very good). The mean rating was $2.8(S D=1.4)$, indicating an average prognosis, although scores were almost equally distributed across the scale.

The parents of medically healthy children were recruited through advertisements in local media. To be eligible for the study, they had to (a) have at least one child aged 1-18 years living at home; (b) match a parent in the cancer group on age, gender, ethnicity, and marital status; (c) have children with a lifetime history free of chronic medical and psychiatric illness; and (d) be free of major stressors, such as divorce, bereavement, unemployment, and family illness during the past year. These eligibility requirements ensured that parents of medically healthy children were experiencing relatively low levels of psychological stress.

\section{Procedures}

All parents attended an initial laboratory session. After providing written informed consent, they completed a short battery of psychosocial instruments (described below). Parents were then seated in a comfortable chair and had $35 \mathrm{ml}$ of blood drawn through antecubital venipuncture. The blood was drawn into heparin-coated Vacutainers (Becton-Dickinson, Franklin Lakes, NJ) and was immediately delivered to the laboratory, where assays began within $2 \mathrm{hr}$. To control for diurnal variations in immune response, we performed all blood draws between 9:00 a.m. and 12:00 p.m.

Over the course of the next 2 days, parents gathered salivary cortisol samples as they went about their normal daily activities. To facilitate this process, we lent them programmable wristwatches (WatchMinder, Irvine, CA) that generated audible beeps five times each day. The beeps cued parents to begin a standard saliva collection protocol that involves chewing on a cotton dental roll (Salivette, Sartstedt Inc., Newton, NC) until it is saturated with saliva. The dental roll is then sealed in a plastic centrifuge tube and refrigerated until it is returned to the laboratory (Kirschbaum \& Hellhammer, 1989).

The watches signaled parents to collect saliva 1, 4, 9, 11, and $13 \mathrm{hr}$ after waking in the morning. Validation studies have shown that this collection schedule yields a fairly robust estimate of total daily cortisol secretion (MacArthur Foundation Network on SES and Health, 2000). To ensure compliance with the schedule, a unique code word appeared on the wristwatch display each time the alarm sounded and disappeared approximately 5 min later. Parents were instructed to copy the code word onto saliva collection containers. Samples with missing or incorrect codes were excluded from analyses. Consistent with previous studies (Ockenfels et al., 1995), approximately $18 \%$ of participants' saliva samples (93/500) were excluded from analyses because they had missing or incorrect codes, were not collected properly, or were not collected at all.

\section{Measuring Psychological States}

Perceived stress. We assessed parents' levels of perceived psychological stress with the 10-item Perceived Stress Scale (PSS; Cohen \& Williamson, 1988). This measure taps the extent to which people find their lives to be unpredictable, uncontrollable, and unmanageable. It covers the previous 1 month. The PSS showed high levels of internal consistency in this sample $(\alpha=.92)$.

Mood states. We assessed mood states using 25 adjectives derived predominately from the Profile of Mood States (McNair, Lorr, \& Droppleman, 1971; Usala \& Hertzog, 1989). These items were combined to form composites reflecting negative affect $(\alpha=.80)$ and positive affect $(\alpha=$ .91) during the previous 1 month.

Depressive symptoms. We assessed parents' depressive symptoms with the Center for Epidemiologic Studies-Depression Scale (Radloff, 
1977). The CES-D is a 20-item measure of the frequency of depressive symptomatology over the past week. It has excellent internal consistency in our sample $(\alpha=.93)$.

Perceived social support. We measured perceived social support with a modified version of the Interpersonal Support Evaluation List (Cohen, Mermelstein, Kamarck, \& Hoberman, 1985). Factor analyses were used to derive two 4-item composites reflecting parents' perceptions of available appraisal support $(\alpha=.84)$ and tangible support $(\alpha=.72)$.

\section{Measuring Health Practices}

We measured health practices using a self-report battery used in our previous work (G. E. Miller et al., 1999; G. E. Miller, Cohen, Rabin, Skoner, \& Doyle, 1999). Participants were classified as smokers if they reported daily use of cigarettes, pipes, or cigars. Alcohol use was determined by counting the number of alcoholic drinks consumed during a typical week. A drink was considered a bottle or can of beer, a glass of wine, or a shot of hard liquor. Regular physical activity was measured with a modified version of the Paffenbarger Activity Scale (Paffenbarger, Blair, Lee, \& Hyde, 1993). This scale provided estimates of typical weekly energy expenditure and the number of minutes of brisk physical activity per week. Sleep hygiene was assessed with the Pittsburgh Sleep Quality Index (Buysse, Reynolds, Monk, Berman, \& Kupfer, 1989). This scale yields estimates of subjective sleep quality (on a 1-4 scale, with higher numbers indicating better sleep quality) and sleep efficiency (the percentage of time in bed actually spent sleeping).

This battery of measures has excellent psychometric properties. Testretest reliability coefficients, assessed over a 6-month period, exceed .70 for all items except subjective sleep quality (G. E. Miller, Cohen, \& Herbert, 1999). With regard to validity, our work with this inventory has shown that clinically depressed patients report greater tobacco use, lower physical activity, and poorer sleep hygiene compared with healthy controls. These health practices also have been linked with in vitro measures of immune response, including reduced natural killer cell cytotoxicity and poorer mitogen-stimulated lymphocyte proliferation (G. E. Miller, Cohen, \& Herbert, 1999; G. E. Miller, Cohen, Rabin, et al., 1999) as well as impaired host resistance to upper respiratory infection (Cohen et al., 1997).

\section{Measuring Daily Patterns of Cortisol Secretion}

After they were returned to the laboratory, salivettes were centrifuged for $5 \mathrm{~min}$ at 3,000 rpm until a clear, low-viscosity supernatant emerged. The supernatants were then collected and frozen at $-70{ }^{\circ} \mathrm{C}$ until the end of the study, at which time they were shipped on dry ice to the Institute of Experimental Psychology II at the University of Düsseldorf, Düsseldorf, Germany. Cortisol assays were performed in duplicate using a timeresolved fluorescence immunoassay with a cortisol-biotin conjugate as a tracer (Kirschbaum, Kudielka, Gaab, Schommer, \& Hellhammer, 1999; Pruessner, Hellhammer, \& Kirschbaum, 1999). This assay has a sensitivity of $0.43 \mathrm{nmol} / \mathrm{L}$. The intra-assay coefficient of variation $(\mathrm{CV})$ was less than $10 \%$. The interassay $\mathrm{CV}$ was less than $12 \%$.

After cortisol values had been log-transformed, each day's data were used to create two indices of secretion for later analysis. The first index was an area-under-the-curve measure reflecting the total volume of cortisol secretion over the day. This was computed using the trapezoidal method, such that higher values reflect greater cortisol release. The second index was a slope reflecting the diurnal pattern of cortisol secretion. This was created by estimating a simple linear regression model for each participant, where his or her cortisol values were regressed on time since waking. Because cortisol secretion generally declines over the day, most healthy individuals have negatively signed diurnal slopes. As a result, lower values, reflecting more rapid declines, are viewed as adaptive. In contrast, higher slope values, as they approach zero, reflect flat diurnal rhythms and are viewed as evidence of HPA dysregulation (McEwen, 1998; Stone et al.,
2001; Yehuda, Teicher, Trestman, Levengood, \& Siever, 1996). ${ }^{3}$ In a very small minority of cases ( 3 parents of cancer patients and 1 parent of a medically healthy child), we observed a daily slope value that exceeded zero, indicating abnormally timed diurnal cortisol surges and/or increasing levels over the day.

To obtain more reliable indices of cortisol secretion, we averaged values derived from the 2 days of ambulatory data collection. The correlation between area-under-the-curve values from the 2 days was $r=.39$, and the correlation between slope values was $r=.22$. Similarly, modest day-to-day stability has been seen in other studies in this area (Ockenfels et al., 1995; Smyth et al., 1997).

\section{Measuring Immune System Glucocorticoid Sensitivity}

To measure glucocorticoid sensitivity, we incubated $1.8 \mathrm{ml}$ of whole blood with $200 \mu \mathrm{l}$ of dexamethasone phosphate dissolved in phosphate buffering solution for $30 \mathrm{~min}$. The final in-well concentrations of dexamethasone were $0 \mathrm{nM}, 10 \mathrm{nM}, 100 \mathrm{nM}$, and $250 \mathrm{nM}$ for assays involving IL- $1 \beta$ and TNF- $\alpha$, and $0 \mathrm{nM}, 10 \mathrm{nM}, 50 \mathrm{nM}$, and $100 \mathrm{nM}$ for assays involving IL-6. We then added $220 \mu \mathrm{l}$ of lipopolysaccharide (LPS) dissolved in phosphate buffering solution to each sample. The final concentration of LPS was $100 \mathrm{ng} / \mathrm{ml}$ for assays involving IL- $1 \beta$ and TNF- $\alpha$ and 3 $\mathrm{ng} / \mathrm{ml}$ for assays involving IL- 6 . The samples were then transferred to 24-well plates and incubated overnight $(16-18 \mathrm{hr})$ at $37{ }^{\circ} \mathrm{C}$ with $5 \% \mathrm{CO}_{2}$. The plates were removed from the incubator and immediately centrifuged for $10 \mathrm{~min}$ at 2,000 rpm. Plasma samples were then aspirated and stored at $-20{ }^{\circ} \mathrm{C}$ until the end of the study. At that time, samples were thawed and cytokine assays were performed in duplicate in a single batch using commercially available ELISA kits (R\&D Systems, Minneapolis, MN). Assay sensitivity was $4 \mathrm{pg} / \mathrm{ml}$ for IL- $1 \beta, 3 \mathrm{pg} / \mathrm{ml}$ for IL-6, and $16 \mathrm{pg} / \mathrm{ml}$ for TNF- $\alpha$. The intra- and interassay coefficients of variation were less than $9 \%$ for all cytokine assays. This is a modified version of a protocol that has been used to examine whether acute bouts of exercise modulate glucocorticoid sensitivity (DeRijk et al., 1996; Smits, Grunberg, Derijk, Sterk, \& Hiemstra, 1998)

\section{Results}

\section{Preliminary Analyses}

Table 1 displays characteristics of the sample. Parents of cancer patients did not differ from parents of medically healthy children with respect to age, gender, ethnicity, marital status, years of education, height, weight, body mass index, over-the-counter medication use, or oral contraceptive use (all $p \mathrm{~s}>.20$ ). The groups did differ with respect to family size, however, with parents of cancer patients having marginally more children living at home, $t(48)=1.92, p<.07$. Statistically controlling for family size, however, did not substantively alter any of the findings we report in the remainder of this article.

\section{Psychological Outcomes}

Table 2 presents psychological outcomes for the groups. Parents of cancer patients reported significantly more psychological dis-

\footnotetext{
${ }^{3}$ Although a circadian decline in cortisol secretion has long been viewed as a stable feature of healthy adults, recent evidence suggests that considerable variability in this pattern exists, both within and across individuals In fact, a substantial minority of adults show a pattern of flat diurnal rhythms, even in the absence of stressful circumstances and/or medical conditions involving the HPA axis (Smyth et al., 1997; Stone et al., 2001).
} 
Table 1

Demographic Characteristics of the Sample

\begin{tabular}{|c|c|c|c|c|c|c|}
\hline \multirow[b]{2}{*}{ Characteristic } & \multicolumn{3}{|c|}{ Parents of children with cancer } & \multicolumn{3}{|c|}{$\begin{array}{l}\text { Parents of medically } \\
\text { healthy children }\end{array}$} \\
\hline & $M$ & $S D$ & Range & $M$ & $S D$ & Range \\
\hline Age (years) & 36.5 & 6.0 & $24-47$ & 37.5 & 5.5 & $28-47$ \\
\hline$\%$ female & 76.0 & & & 76.0 & & \\
\hline$\%$ Caucasian & 88.0 & & & 88.0 & & \\
\hline$\%$ married & 80.0 & & & 80.0 & & \\
\hline No. of children living at home & 2.5 & 1.3 & $1-6$ & 1.9 & 1.1 & $1-6$ \\
\hline Education (years) & 15.2 & 2.7 & $9-20$ & 15.5 & 2.2 & $12-18$ \\
\hline Height (m) & 1.7 & 0.1 & $1.6-1.9$ & 1.7 & 0.0 & $1.5-1.9$ \\
\hline Weight $(\mathrm{kg})$ & 78.2 & 28.1 & $49-172$ & 70.2 & 15.2 & $51-104$ \\
\hline Body mass index $\left(\mathrm{kg} / \mathrm{m}^{2}\right)$ & 27.0 & 7.5 & $17-46$ & 24.7 & 5.2 & $19-40$ \\
\hline$\%$ using OTC medications & 48.0 & & & 56.0 & & \\
\hline$\%$ using oral contraceptives & 20.0 & & & 32.0 & & \\
\hline
\end{tabular}

Note. $n=25$ in each group. OTC $=$ over the counter.

tress than parents of medically healthy children. They described higher levels of perceived stress, $t(48)=2.03, p<.05$; greater overall negative affect, $t(48)=2.38, p<.03$; less overall positive affect, $t(48)=-3.04, p<.01$; and significantly more frequent depressive symptoms, $t(48)=3.44, p<.01$.

\section{Baseline Cytokine Production}

We next examined whether chronic stress influenced the extent of parents' baseline cytokine production. In the LPS-stimulated cultures treated with saline (but not dexamethasone), parents of cancer patients exhibited lower average levels of IL-6, greater average levels of TNF- $\alpha$, and no differences in IL- $1 \beta$, compared with parents of healthy children. None of these differences, however, approached statistical significance ( $p$ s $>.13$ ). The $M \pm S D$ values for parents of cancer patients were $733 \pm 820,1,110 \pm 881$, and $1,358 \pm 1,001$ for IL- 6 , TNF- $\alpha$, and IL- $1 \beta$, respectively. The corresponding values for parents of medically healthy children were $1,134 \pm 783,800 \pm 407$, and 1,305 \pm 672 .

\section{Immune System Glucocorticoid Sensitivity}

We next explored whether chronic stress influenced the immune system's sensitivity to the anti-inflammatory actions of glucocor-

Table 2

Perceived Stress, Mood States, and Depressive Symptoms

\begin{tabular}{|c|c|c|c|c|}
\hline \multirow[b]{2}{*}{ Characteristic } & \multicolumn{2}{|c|}{$\begin{array}{l}\text { Parents of } \\
\text { children with } \\
\text { cancer }\end{array}$} & \multicolumn{2}{|c|}{$\begin{array}{c}\text { Parents of } \\
\text { medically } \\
\text { healthy children }\end{array}$} \\
\hline & $M$ & $S D$ & $M$ & $S D$ \\
\hline Perceived stress & $18.3_{\mathrm{a}}$ & 8.2 & $13.8_{\mathrm{b}}$ & 7.6 \\
\hline Negative affect & $25.6_{\mathrm{a}}$ & 9.8 & $19.6_{\mathrm{b}}$ & 7.7 \\
\hline Positive affect & $19.3_{\mathrm{a}}$ & 5.5 & $23.7_{\mathrm{b}}$ & 4.7 \\
\hline Depressive symptoms & $16.8_{\mathrm{a}}$ & 12.6 & $7.2_{\mathrm{b}}$ & 6.0 \\
\hline
\end{tabular}

Note. $n=25$ in each group. For each construct, means with different subscripts indicate group differences at $p<.05$ by independent samples $t$ test. ticoids. This was done using a series of repeated-measures analyses of variance, with group (parents of cancer patients vs. parents of healthy children) and dosage (low vs. medium vs. high dexamethasone concentration) serving as the independent variables. To control for variability in the extent of baseline cytokine production, we included in these analyses cytokine values from the salinetreated cultures as covariates. Average values for each cytokine appear in Table 3.

$I L-1 \beta$. There were no reliable differences in dexamethasone's capacity to suppress IL- $1 \beta$ production between parents of cancer patients and parents of medically healthy children, $F(1$, $35)=1.08, p>.31$. A significant main effect of dosage emerged, however, indicating that IL- $1 \beta$ production declined with greater concentrations of dexamethasone, $F(2,34)=159.68, p<.01$. The Group $\times$ Dosage interaction was nonsignificant, $F(2,34)=1.24$, $p>.29$. These findings are illustrated in Figure 1 .

IL-6. Parents of cancer patients showed significantly less dexamethasone suppression of IL-6 production compared with parents of medically healthy children, $F(1,40)=4.12, p<.05$ This pattern of findings was consistent across dosages of dexamethasone as indicated by the nonsignificant Group $\times$ Dosage interaction, $F(2,39)=2.45, p>.10$. A main effect of dosage emerged in this analysis, with IL-6 production declining with higher concentrations of dexamethasone, $F(2,39)=88.54, p<$ .01 . These results are illustrated in Figure 2.

$T N F-\alpha$. There were no significant differences in dexamethasone-related suppression of TNF- $\alpha$ production between parents of cancer patients and parents of medically healthy children, $F(1$, $45)=0.01, p>.95$. There was also no significant Group $\times$ Dosage interaction, $F(2,44)=0.71, p>.49$. A reliable main effect of dosage was found, with TNF- $\alpha$ production declining in the presence of greater concentrations of dexamethasone, $F(2$, $44)=439.92, p<.01$. These findings are illustrated in Figure 3

\section{Depression, Health Practices, and Cortisol as Mediators}

Next, we examined whether depressive symptoms, health practices, or cortisol secretion might operate as pathways linking chronic stress with declines in IL-6 glucocorticoid sensitivity. Standard procedures require that data meet three criteria to provide 
Table 3

Mean Pro-Inflammatory Cytokine Values

\begin{tabular}{|c|c|c|c|c|}
\hline \multirow[b]{2}{*}{ Cytokine and dexamethasone dosage } & \multicolumn{2}{|c|}{$\begin{array}{l}\text { Parents of children } \\
\text { with cancer }\end{array}$} & \multicolumn{2}{|c|}{$\begin{array}{l}\text { Parents of medically } \\
\text { healthy children }\end{array}$} \\
\hline & $M$ & $S D$ & $M$ & $S D$ \\
\hline \multicolumn{5}{|l|}{ Interleukin- $1 \beta$} \\
\hline $10 \mathrm{nM}$ dexamethasone & 1,111 & 301 & 1,002 & 327 \\
\hline $100 \mathrm{nM}$ dexamethasone & 317 & 127 & 318 & 136 \\
\hline $250 \mathrm{nM}$ dexamethasone & 167 & 115 & 182 & 123 \\
\hline \multicolumn{5}{|l|}{ Interleukin-6 } \\
\hline $10 \mathrm{nM}$ dexamethasone & 1,036 & 291 & 830 & 309 \\
\hline $100 \mathrm{nM}$ dexamethasone & 545 & 152 & 419 & 161 \\
\hline $250 \mathrm{nM}$ dexamethasone & 351 & 165 & 163 & 174 \\
\hline \multicolumn{5}{|l|}{ Tumor necrosis factor- $\alpha$} \\
\hline $10 \mathrm{nM}$ dexamethasone & 853 & 134 & 878 & 129 \\
\hline $100 \mathrm{nM}$ dexamethasone & 535 & 106 & 522 & 101 \\
\hline $250 \mathrm{nM}$ dexamethasone & 353 & 96 & 354 & 91 \\
\hline
\end{tabular}

Note. Cytokine production following stimulation with lipopolysaccharide $(100 \mathrm{ng} / \mathrm{ml}$ for assays involving interleukin- $1 \beta$ and tumor necrosis factor- $\alpha$ and $3 \mathrm{ng} / \mathrm{ml}$ for assays involving interleukin-6). Values are adjusted for baseline cytokine production, that is, in a culture in which no dexamethasone is added. All values are in picograms per milliliter.

evidence consistent with a mediational hypothesis: (a) The predictor variable (group membership) must be related to the outcome variable (glucocorticoid sensitivity), (b) the predictor variable must be related to the hypothesized mediator (variables representing depressive symptoms, health practices, and cortisol secretion), and (c) the magnitude of the relationship between the predictor and outcome variables must be substantially reduced when the mediator is statistically controlled (Stone, 1992). At the outset we should note that these methods cannot provide a strong test of whether the mediators operate in a causal fashion. Such a test would require an experimental manipulation. What they can provide, however, is an indication of whether each mechanism relates to chronic stress and IL-6 glucocorticoid sensitivity in a way that is consistent with a mediational hypothesis.

Depressive symptoms. Parents of cancer patients, as we noted earlier, described more frequent depressive symptoms than parents of medically healthy children. Depressive symptoms were unrelated to glucocorticoid sensitivity, $r(40)=-.05, p>.76$, however, and statistically controlling for them did not appreciably reduce

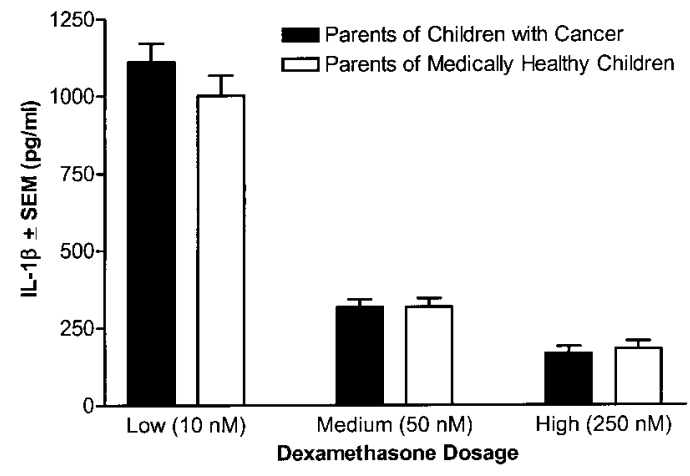

Figure 1. Chronic stress and dexamethasone's capacity to suppress in vitro interleukin-1 $\beta$ (IL-1 $\beta$ ) production. Values are adjusted for baseline cytokine production. SEM $=$ standard error of the mean. group differences in IL-6 glucocorticoid sensitivity $(<2 \%$ reduction in variance accounted for by group). These findings suggest that depressive symptoms were not responsible for the decline in IL-6 glucocorticoid sensitivity among parents of cancer patients. ${ }^{4}$

Health practices. Table 4 presents health practices for the sample. Parents of cancer patients reported significantly worse sleep quality than parents of medically healthy children, $t(48)=2.65, p<.02$. They also reported marginally less efficient sleep, $t(48)=-1.76, p<.09$. The two groups did not differ with respect to smoking status, alcohol consumption, minutes per week of brisk physical activity, or weekly energy expenditure (all $p \mathrm{~s}>$ .18). Statistically controlling for the two health practices that differentiated the groups, sleep quality and sleep efficiency, did not appreciably reduce disparities in IL-6 glucocorticoid sensitivity $(<5 \%$ reduction in total variance accounted for), nor did controlling for the other health practices we assessed or height, weight, body mass index, over-the-counter medication use, or oral contraceptive use $(<5 \%$ reduction in variance accounted for by group). These findings suggest that health practices, anthropometric characteristics, and medication use were not responsible for the reduction in glucocorticoid sensitivity among parents of cancer patients.

Cortisol secretion. Figure 4 displays parents' diurnal patterns of cortisol secretion. Parents of children with cancer showed significantly flatter diurnal slopes than parents of medically healthy children $(M=-.046, S D=.02$ vs. $M=-.058, S D=.02)$, $t(44)=3.11, p<.01$. The flattening was due to a significant reduction in cortisol secretion at $1 \mathrm{hr}$ postawakening among parents of cancer patients, $t(44)=-2.94, p<.01$. The groups did not

\footnotetext{
${ }^{4}$ These mediational analyses use aggregate IL-6 production as an outcome. In this index, values are collapsed across low, medium, and high doses of dexamethasone after baseline IL- 6 production has been covaried out. The same strategy is used in the next section, in which we examine the buffering role of social support. Identical results emerge when analyses are computed separately for each dosage.
} 


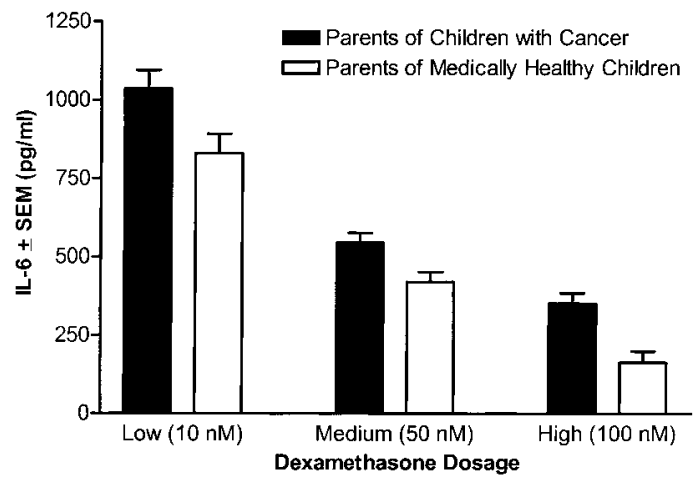

Figure 2. Chronic stress and dexamethasone's capacity to suppress in vitro interleukin-6 (IL-6) production. Values are adjusted for baseline cytokine production. SEM $=$ standard error of the mean.

differ with respect to cortisol concentration at any other time of the day $(t \mathrm{~s}<0.50, p \mathrm{~s}>.62)$. No group differences emerged for the area-under-curve measure reflecting total daily volume of cortisol secretion $(M=10.61, S D=1.62$ vs. $M=11.08, S D=1.93)$, $t(44)=-1.10, p>.28$.

Despite these findings, cortisol slopes were unrelated to glucocorticoid sensitivity, $r(40)=-.05, p>.76$, and statistically controlling for them did not appreciably reduce group differences in IL-6 glucocorticoid sensitivity $(<5 \%$ reduction in variance accounted for by group). The same was true of cortisol secretion at $1 \mathrm{hr}$ postawakening, $r(40)=-.20, p>.23(<5 \%$ reduction in total variance accounted for). These findings suggest that diurnal patterns of cortisol secretion were not responsible for the decline in IL-6 glucocorticoid sensitivity among parents of cancer patients.

\section{Social Support as a Stress Buffer}

To determine whether social support buffered parents of cancer patients from declines in IL-6 glucocorticoid sensitivity, we computed a series of multiple regression equations where sensitivity was predicted by group (parents of cancer patients vs. parents of medically healthy children), social support (either appraisal or tangible support scores), and a product term representing the interaction of these variables (Aiken \& West, 1991). A significant interaction between group membership and tangible social support emerged (for interaction term, $\Delta R^{2}=.01, b=.46$ ) $t(43)=2.04$, $p<.05$. Tangible social support was unrelated to glucocorticoid sensitivity among parents of medically healthy children (simple slope $=.01$ ). Among parents of cancer patients, however, glucocorticoid sensitivity declined (i.e., resistance increased) to the extent that parents reported low tangible support (simple slope $=$ -.05). These findings suggest that tangible support buffered participants from the reduction in IL-6 glucocorticoid sensitivity that accompanies caring for a child with cancer. No evidence of a buffering effect was detected for appraisal support (for interactions term, $p>.40$ ). The parent groups did not differ with respect to mean levels of either tangible or appraisal support ( $p s>.25$ ).

\section{Discussion}

This study's primary hypothesis was that chronic psychological stress would impair the immune system's capacity to respond to the anti-inflammatory actions of glucocorticoid hormones. It yielded preliminary support for this hypothesis. Among parents of cancer patients, dexamethasone's capacity to suppress IL-6 production was significantly reduced compared with parents of medically healthy children. These findings are consistent with recent studies demonstrating that the immune system's glucocorticoid sensitivity declines following short bouts of physical activity and acute stress in humans (DeRijk et al., 1996; Rohleder, Schommer, Hellhammer, Engel, \& Kirschbaum, 2001; Smits et al., 1998) and following intermittent bouts of social disruption stress in mice (Avitsur, Stark, \& Sheridan, 2001; Stark et al., 2001). Collectively, these findings suggest a novel mechanism through which psychological stress could influence the onset and/or progression of conditions that involve excessive inflammation. This is the case in many disease contexts, including allergic, autoimmune, cardiovascular, infectious, and rheumatologic illnesses. A stress-induced immunosuppression model, as we mentioned earlier, cannot provide a parsimonious explanation for how such a process might occur.

This study also explored pathways linking chronic stress with reductions in IL-6 glucocorticoid sensitivity. In terms of affective pathways, parents of cancer patients reported more frequent depressive symptoms than parents of healthy children, but subsequent analyses suggested that depression did not operate as a mediator. It is possible that chronic stress contributes to declines in immune system glucocorticoid sensitivity through other affective pathways (e.g., anxiety) or through nonaffective pathways, such as the formation of intrusive thoughts or feelings of helplessness. In terms of health practices, the groups did not differ with respect to smoking status, alcohol consumption, or physical activity. Although parents of cancer patients reported worse sleep hygiene than parents of healthy children, mediational analyses suggested that sleep was not responsible for stress-related reductions in immune system glucocorticoid sensitivity. Group differences also emerged in daily patterns of cortisol secretion. Parents of cancer patients had significantly flatter diurnal slopes than parents of medically healthy children, primarily because of a reduced output at $1 \mathrm{hr}$ postawakening. Disparities in cortisol secretion patterns, however, could not explain the reduction in IL-6 glucocorticoid sensitivity among parents of cancer patients.

So, what mechanisms might be responsible for this effect? One possibility is that hormonal products of the SAM axis were oper-

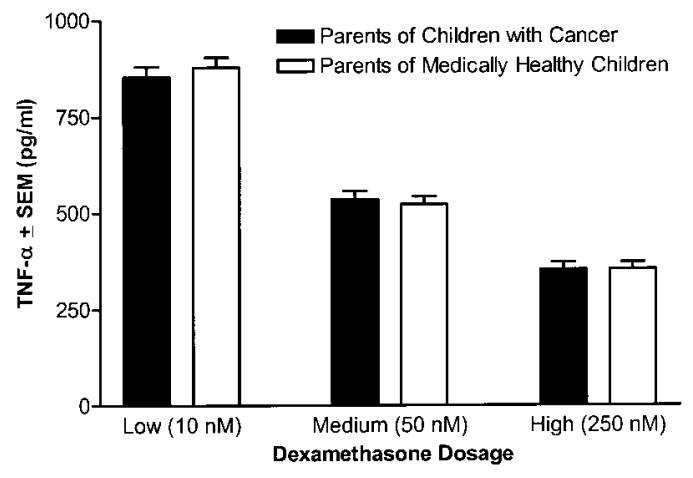

Figure 3. Chronic stress and dexamethasone's capacity to suppress in vitro tumor necrosis factor- $\alpha$ (TNF- $\alpha)$ production. Values are adjusted for baseline cytokine production. SEM $=$ standard error of the mean. 
Table 4

Health Practices

\begin{tabular}{|c|c|c|c|c|}
\hline \multirow[b]{2}{*}{ Practice } & \multicolumn{2}{|c|}{$\begin{array}{l}\text { Parents of children } \\
\text { with cancer }\end{array}$} & \multicolumn{2}{|c|}{$\begin{array}{l}\text { Parents of medically } \\
\text { healthy children }\end{array}$} \\
\hline & $M$ & $S D$ & $M$ & $S D$ \\
\hline \multicolumn{5}{|l|}{ Tobacco and alcohol consumption } \\
\hline$\%$ current smokers & 20.0 & & 12.0 & \\
\hline$\%$ former smokers & 8.0 & & 8.0 & \\
\hline Alcoholic drinks (per week) & 2.3 & 6.1 & 1.9 & 3.1 \\
\hline \multicolumn{5}{|l|}{ Physical activity } \\
\hline Brisk activity (min/week) & 46.0 & 72.9 & 80.2 & 103.5 \\
\hline Energy expenditure (Kcal/week) & 875.1 & 659.8 & $1,193.9$ & $1,058.2$ \\
\hline \multicolumn{5}{|l|}{ Sleep hygiene } \\
\hline Subjective sleep quality (1-4) & $2.8 \mathrm{a}$ & 1.4 & $3.1_{\mathrm{b}}$ & 1.4 \\
\hline Sleep efficiency & $0.8^{\mathrm{a}}$ & 0.1 & $0.9^{\circ}$ & 0.1 \\
\hline
\end{tabular}

Note. $n=25$ in each group. For each construct, means with different subscripts indicate group differences at $p<.05$ by independent samples $t$ test.

ating as mediators. To the extent that chronic stress triggered the secretion of epinephrine and norepinephrine, these hormones could have downregulated glucocorticoid receptor expression and thereby contributed to the diminished glucocorticoid sensitivity of IL-6 (DeRijk et al., 1996; Maccari et al., 1992). Future studies might evaluate this hypothesis by collecting information regarding long-term epinephrine and norepinephrine output (Baum \& Grunberg, 1995). It is also possible that cortisol was responsible for the reduced IL-6 glucocorticoid sensitivity among parents of cancer patients but did not emerge as a mediator in our study because of the timing of measurements. The HPA axis habituates to stressful experience fairly rapidly, and, in some cases, cortisol secretion rebounds to below normal levels (Frankenhauser, 1975; Heim, Ehlert, \& Hellhammer, 2000; Lundberg, 1980). It is interesting to note that this pattern of blunted secretion emerged in an early study with parents of cancer patients (Friedman, Mason, \& Hamburg, 1963), which found that cortisol levels were remarkably stable across time, even during periods when a child's medical status had deteriorated significantly. Blunted cortisol secretion also has been described in patients who suffer from posttraumatic stress disorder (Yehuda, 1998, 2000; Yehuda et al., 1996), teachers with workrelated burnout (Pruessner et al., 1999), women with conflicting role demands (Adam \& Gunnar, 2001), breast cancer patients who

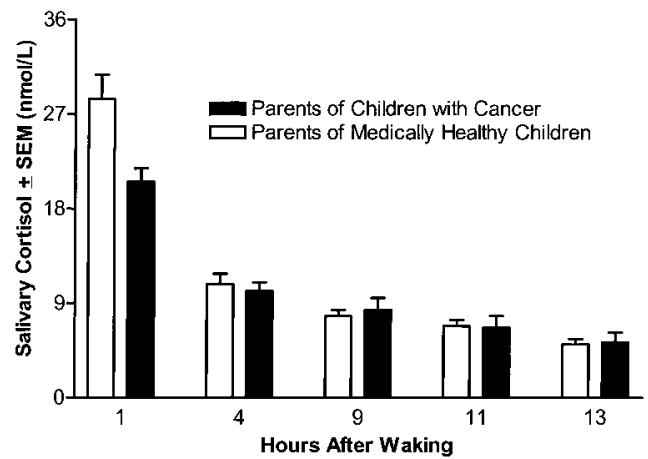

Figure 4. Diurnal patterns of cortisol secretion. SEM $=$ standard error of the mean. experience accelerated mortality (Sephton, Sapolsky, Kraemer, \& Spiegel, 2000), and soldiers in the midst of combat (Bourne, Rose, \& Mason, 1967, 1968). Given that the average parent in our study had been dealing with cancer for more than 9 months at the time he or she participated, it is conceivable that declines in IL-6 glucocorticoid sensitivity arose from exposure to high concentrations of cortisol in the months shortly after diagnosis. With the passage of time, HPA axis function may have rebounded, while glucocorticoid receptor expression and/or function within white blood cells remained downregulated. To properly evaluate this hypothesis, of course, parents of cancer patients would need to be studied longitudinally from the time of diagnosis. Finally, it is possible that cortisol was responsible for chronic stress-related declines in IL-6 glucocorticoid sensitivity but did not emerge as a mediator because of the wide variability in secretion patterns across days. Future studies might overcome this reliability problem by boosting enrollment figures, collecting samples more frequently, and/or increasing the number of days of saliva collection (Stone et al., 2001).

We also hypothesized that social support would operate in a buffering fashion. Clear support emerged for this hypothesis, as support was unrelated to glucocorticoid sensitivity among parents of medically healthy children. Among parents of cancer patients, however, IL-6 glucocorticoid sensitivity declined to the extent that parents reported low tangible support. These findings suggest that the provision of material aid can offset the immunologic consequences of caring for a child with cancer, perhaps by ameliorating the substantial economic, occupational, and familial disruptions imposed by the disease and its treatment. Although it is not clear why, appraisal support does not appear to have the same impact on glucocorticoid sensitivity. Nevertheless, our findings corroborate evidence (Baron et al., 1990; Glaser et al., 1985, 1992; KiecoltGlaser et al., 1991; Kiecolt-Glaser, Garner, et al., 1984; KiecoltGlaser, Speicher, et al., 1984) suggesting that social support has the capacity to buffer people from the immunologic consequences of chronically stressful experience.

It is unclear why chronic stress did not also diminish dexamethasone's capacity to suppress IL- $1 \beta$ and TNF- $\alpha$. Glucocorticoids can block the production of all three cytokines we studied by 
inducing transcription of $\mathrm{I} \kappa \mathrm{B}$. This molecule prevents NF- $\kappa \mathrm{B}$ from translocating to the cell nucleus, where it activates the genes responsible for pro-inflammatory cytokine production (Scheinman, Cogswell, Lofquist, \& Baldwin, 1995). Hence, there is no clear signaling-pathway explanation for the differential effects we observed. Other studies have found that short bouts of exercise and stress can reduce the glucocorticoid sensitivity of IL-1 $\beta$ and TNF- $\alpha$ (DeRijk et al., 1996, 1997; Rohleder et al., 2001; Smits et al., 1998). In fact, some evidence indicates that the sensitivity of these cytokines declines to a greater extent than IL-6 (DeRijk et al., 1997). Although the reason for these discrepancies is unclear, it seems likely that they are related to different neural and/or endocrine pathways being activated during acute versus chronic and psychological versus physiological forms of stress.

This study had a number of limitations. Perhaps the most important was its cross-sectional design, which precludes us from making any causal inferences regarding the relationship between chronic psychological stress and IL-6 glucocorticoid sensitivity. Although reverse causality is an unlikely explanation for our findings, it is conceivable that some unmeasured third variable was responsible, such as exposure to an environmental toxin that increases offspring cancer risk and disrupts immune function. Another important limitation of the study was that it relied on an assay system that does not closely resemble the in vivo environment in which white blood cells operate. Findings would have been more compelling if cortisol, rather than dexamethasone, had been used to suppress the production of cytokines. Although both of these agents have anti-inflammatory properties, dexamethasone is nearly 30 times more potent and primarily operates through a different isoform of the glucocorticoid receptor (Wilckens \& DeRijk, 1997). Future studies may resolve this problem, and enhance their chances of detecting subtle alterations in glucocorticoid sensitivity, by using a cortisol-based assay system. Finally, it is not clear what implications our findings have for in vivo immune system glucocorticoid sensitivity. The stress-related declines in IL-6 sensitivity we observed were relatively small in magnitude, occurred in peripheral white blood cells stimulated in vitro, and did not extend to the other cytokines we assessed. These issues place important constraints on the generalizability of our findings to in vivo circumstances.

This study suggests that chronic psychological stress may reduce the immune system's sensitivity to hormonal signals that normally terminate the inflammatory cascade. Before any definitive conclusions about this process can be reached, however, studies will need to replicate these findings and determine whether this represents a truly causal phenomenon. The latter could be accomplished through an experimental intervention trial aimed at increasing tangible support for parents of cancer patients (G. E. Miller \& Cohen, 2001). It will also be important for future studies to delineate the mechanisms responsible for IL-6 glucocorticoid sensitivity, perhaps by longitudinally studying psychological, hormonal, and immune processes in parents of cancer patients from the time of their child's initial diagnosis. A study of this nature would also enable investigators to explore the psychobiological consequences of dysregulated cytokine production. Given accumulating evidence that pro-inflammatory cytokines operate as stimulus for HPA activation (Maier \& Watkins, 1998; A. H. Miller et al., 1999) and may contribute to depressive symptoms (Capuron,
Ravaud, \& Dantzer, 2000; Musselman et al., 2001), this could be a very fruitful line of research.

\section{References}

Adam, E. K., \& Gunnar, M. R. (2001). Relationship functioning and home and work demands predict individual differences in the diurnal cycle of cortisol in women. Psychoneuroendocrinology, 26, 189-208.

Aiken, L. S., \& West, S. G. (1991). Multiple regression: Testing and interpreting interactions. London: Sage.

American Psychiatric Association. (1994). Diagnostic and statistical manual of mental disorders (4th ed.). Washington, DC: Author.

Andersen, B. L., Kiecolt-Glaser, J. K., \& Glaser, R. (1994). A biobehavioral model of cancer stress and disease course. American Psychologist, 49, 389-404.

Avitsur, R., Stark, J. L., \& Sheridan, J. F. (2001). Social stress induces glucocorticoid resistance in animals. Hormones and Behavior, 39, 247 257.

Baron, R. S., Cutrona, C. E., Hicklin, D., Russell, D. W., \& Lubaroff, D. M. (1990). Social support and immune function among spouses of cancer patients. Journal of Personality and Social Psychology, 59, $344-352$.

Baum, A., \& Grunberg, N. (1995). Measurement of stress hormones. In S. Cohen, R. C. Kessler, \& L. G. Underwood (Eds.), Measuring stress: A guide for health and social scientists (pp. 193-212). New York: Oxford University Press.

Blalock, J. E. (1994). The syntax of neuroendocrine-immune communication. Immunology Today, 15, 504-511.

Bourne, P. G., Rose, R. M., \& Mason, J. W. (1967). Urinary 17-OCHS levels: Data on seven helicopter ambulance medics in combat. Archives of General Psychiatry, 17, 104-110.

Bourne, P. G., Rose, R. M., \& Mason, J. W. (1968). 17-OCHS levels in combat: Special forces A-Team under threat of attack. Archives of General Psychiatry, 19, 135-140.

Buysse, D. J., Reynolds, C. F., Monk, T. H., Berman, S. R., \& Kupfer, D. J. (1989). The Pittsburgh Sleep Quality Index: A new instrument for psychiatric practice and research. Psychiatry Research, 28, 193-213.

Capuron, L., Ravaud, A., \& Dantzer, R. (2000). Early depressive symptoms in cancer patients receiving interleukin-2 and/or interferon alfa- $2 b$ therapy. Journal of Clinical Oncology, 18, 2143-2151.

Chrousos, G. P. (1995). The hypothalamic-pituitary-adrenal axis and immune mediated inflammation. New England Journal of Medicine, 332, 1351-1362.

Chrousos, G. P., Detera-Wadleigh, S. D., \& Karl, M. (1993). Syndromes of glucocorticoid resistance. Annals of Internal Medicine, 119, 1113-1124.

Cohen, S., Doyle, W. J., Skoner, D. P., Rabin, B. S., \& Gwaltney, J. M., Jr. (1997). Social ties and susceptibility to the common cold. Journal of the American Medical Association, 277, 1940-1944.

Cohen, S., Frank, E., Doyle, W. J., Skoner, D. P., Rabin, B. S., \& Gwaltney, J. M., Jr. (1998). Types of stressors that increase susceptibility to the common cold in healthy adults. Health Psychology, 17, 214-223.

Cohen, S., Mermelstein, R., Kamarck, T. W., \& Hoberman, H. (1985). Measuring the functional components of social support. In I. G. Sarason \& B. R. Sarason (Eds.), Social support: Theory, research, and application (pp. 73-94). The Hague, Netherlands: Martinus Nijhoff.

Cohen, S., Miller, G. E., \& Rabin, B. S. (2001). Psychological stress and antibody response to immunization: A critical review of the human literature. Psychosomatic Medicine, 63, 7-18.

Cohen, S., Tyrrell, D. A., \& Smith, A. P. (1991). Psychological stress and susceptibility to the common cold. New England Journal of Medicine, 325, 606-612.

Cohen, S., \& Williamson, G. M. (1988). Perceived stress in a probability sample of the United States. In S. Spacapan \& S. Oskamp (Eds.), The social psychology of health (pp. 31-67). Newbury Park, CA: Sage. 
Cohen, S., \& Williamson, G. M. (1991). Stress and infectious disease in humans. Psychological Bulletin, 109, 5-24.

DeRijk, R., Michelson, D., Karp, B., Petrides, J., Galliven, E., Deuster, P., et al. (1997). Exercise and circadian rhythm-induced variations in plasma cortisol differentially regulate interleukin-1 beta (IL-1 beta), IL-6, and tumor necrosis factor-alpha (TNF alpha) production in humans: High sensitivity of TNF alpha and resistance of IL-6. Journal of Clinical Endocrinology \& Metabolism, 82, 2182-2191.

DeRijk, R., Petrides, J., Deuster, P., Gold, P. W., \& Sternberg, E. M. (1996). Changes in corticosteroid sensitivity of peripheral blood lymphocytes after strenuous exercise in humans. Journal of Clinical Endocrinology \& Metabolism, 81, 228-235.

DeRijk, R., \& Sternberg, E. M. (1997). Corticosteroid resistance and disease. Annals of Medicine, 29, 79-82.

Felten, S. Y., \& Felten, D. (1994). Neural-immune interaction. Progress in Brain Research, 100, 157-162.

Frankenhauser, M. (1975). Experimental approaches to the study of catecholamines. In L. Levi (Eds.), Emotions-Their parameters and measurement (pp. 209-234). New York: Raven.

Friedman, S. B., Mason, J. W., \& Hamburg, D. A. (1963). Urinary 17-hydroxycorticosteroid levels in parents of children with neoplastic disease. Psychosomatic Medicine, 25, 364-376.

Glaser, R., Kiecolt-Glaser, J. K., Bonneau, R. H., Malarkey, W. B., Kennedy, S., \& Hughes, J. (1992). Stress-induced modulation of the immune response to recombinant hepatitis B vaccine. Psychosomatic Medicine, 54, 22-29.

Glaser, R., Kiecolt-Glaser, J. K., Speicher, C. E., \& Holliday, J. E. (1985). Stress, loneliness, and changes in herpes virus latency. Journal of Behavioral Medicine, 8, 249-260.

Gold, P. W., Goodwin, F. K., \& Chrousos, G. P. (1988a). Clinical and biochemical manifestations of depression: Relation to the neurobiology of stress (Part I). New England Journal of Medicine, 319, 348-353.

Gold, P. W., Goodwin, F. K., \& Chrousos, G. P. (1988b). Clinical and biochemical manifestations of depression: Relation to the neurobiology of stress (Part II). New England Journal of Medicine, 319, 413-420.

Grant, I. (1993). Psychosomatic-somatopsychic aspects of multiple sclerosis. In U. Halbreich (Ed.), Multiple sclerosis: A neuropsychiatric disorder (pp. 119-136). Washington, DC: American Psychiatric Association.

Heim, C., Ehlert, U., \& Hellhammer, D. (2000). The potential role of hypocortisolism in the pathophysiology of stress-related bodily disorders. Psychoneuroendocrinology, 25, 1-35.

Herbert, T. B., \& Cohen, S. (1993). Stress and immunity in humans: A meta-analytic review. Psychosomatic Medicine, 55, 364-379.

Kamarck, T. W., Manuck, S. B., \& Jennings, J. R. (1990). Social support reduces cardiovascular reactivity to psychological challenge: A laboratory model. Psychosomatic Medicine, 52, 42-58.

Kaplan, J. R., Manuck, S. B., Clarkson, T. B., Lusso, F. M., Taub, D. T., \& Miller, E. W. (1983, May 13). Social stress and atherosclerosis in normocholesterolemic monkeys. Science, 220, 733-735.

Kiecolt-Glaser, J. K., Dura, J. R., Speicher, C. E., Trask, O. J., \& Glaser, R. (1991). Spousal caregivers of dementia victims: Longitudinal changes in immunity and health. Psychosomatic Medicine, 53, 345-362.

Kiecolt-Glaser, J. K., Garner, W., Speicher, C. E., Penn, G., Holliday, J. E., \& Glaser, R. (1984). Psychosocial modifiers of immunocompetence in medical students. Psychosomatic Medicine, 46, 7-14.

Kiecolt-Glaser, J. K., \& Glaser, R. (1988). Methodological issues in behavioral immunology research with humans. Brain, Behavior, and Immunity, 2, 67-78.

Kiecolt-Glaser, J. K., Glaser, R., Gravenstein, S., Malarkey, W. B., \& Sheridan, J. F. (1996). Chronic stress alters the immune response to influenza virus vaccine in older adults. Proceedings of the National Academy of Sciences, USA, 93, 3043-3047.

Kiecolt-Glaser, J. K., Marucha, P. T., Malarkey, W. B., Mercado, A. M., \&
Glaser, R. (1995). Slowing of wound healing by psychological stress. Lancet, 346, 1194-1196.

Kiecolt-Glaser, J. K., Speicher, C. E., Holliday, J. E., \& Glaser, R. (1984). Stress and the transformation of lymphocytes by Epstein-Barr virus. Journal of Behavioral Medicine, 7, 1-12.

Kirschbaum, C., \& Hellhammer, D. (1989). Salivary cortisol in psychobiological research: An overview. Neuropsychobiology, 22, 150-169.

Kirschbaum, C., Klauer, T., Sigrun-Heide, F., \& Hellhammer, D. (1995). Sex-specific effects of social support on cortisol and subjective responses to acute psychological stress. Psychosomatic Medicine, 57, 23-31.

Kirschbaum, C., Kudielka, B. M., Gaab, J., Schommer, N. C., \& Hellhammer, D. H. (1999). Impact of gender, menstrual cycle phase, and oral contraceptives on the activity of the hypothalamic-pituitary-adrenal axis. Psychosomatic Medicine, 61, 154-162.

Leung, D. Y. M., De Castro, M., Szefler, S. J., \& Chrousos, G. P. (1998). Mechanisms of glucocorticoid-resistant asthma. Annals of the New York Academy of Sciences, 840, 735-746.

Lundberg, U. (1980). Catecholamine and cortisol excretion under psychologically different experimental conditions. In E. Usdin, R. Kvetnansky, \& I. J. Kopin (Eds.), Catecholamines and stress: Recent advances (pp. 455-480). New York: Elsevier.

MacArthur Foundation Network on SES and Health. (2000, June). Salivary cortisol measurement. San Francisco: Author.

Maccari, S., Mormede, P., Piazza, P. V., Simon, H., Angelucci, L., \& Le Moal, M. (1992). Hippocampal Type 1 and Type 2 corticosteroid receptors are modulated by central noradrenergic systems. Psychoneuroendocrinology, 17, 103-112.

Maier, S. F., \& Watkins, L. R. (1998). Cytokines for psychologists: Implications of bidirectional immune-to-brain communication for understanding behavior, mood, and cognition. Psychological Review, 105, 83-107.

Manne, S. L., Du Hamel, K., Gullelli, K., Sorgen, K., \& Redd, W. H. (1998). Post-traumatic stress disorder among mothers of pediatric cancer survivors: Diagnosis, comorbidity, and utility of the PTSD checklist as a screening instrument. Journal of Pediatric Psychology, 23, 357-366.

Marucha, P. T., Kiecolt-Glaser, J. K., \& Favagehi, M. (1998). Mucosal wound healing is impaired by examination stress. Psychosomatic Medicine, 60, 362-365.

McEwen, B. S. (1998). Protective and damaging effects of stress mediators. New England Journal of Medicine, 338, 171-179.

McNair, D. M., Lorr, M., \& Droppleman, L. F. (1971). Profile of Mood States. San Diego, CA: Education and Industrial Publishing Service.

Miller, A. H., Pariante, C. M., \& Pearce, B. D. (1999). Effects of cytokines on glucocorticoid receptor expression and function. Advances in Experimental Medicine and Biology, 461, 107-116.

Miller, G. E., \& Cohen, S. (2001). Psychological interventions and the immune system: A meta-analytic review and critique. Health Psychology, 20, 47-63.

Miller, G. E., Cohen, S., \& Herbert, T. B. (1999). Pathways linking major depression and immunity in ambulatory female patients. Psychosomatic Medicine, 61, 850-860.

Miller, G. E., Cohen, S., Rabin, B. S., Skoner, D. P., \& Doyle, W. J. (1999). Personality and tonic cardiovascular, neuroendocrine, and immune parameters. Brain, Behavior, and Immunity, 13, 109-123.

Musselman, D. L., Lawson, D. H., Gumnick, J. F., Manatunga, A. K., Penna, S., Goodkin, R. S., et al. (2001). Paroxetine for the prevention of depression induced by high-dose interferon alfa. New England Journal of Medicine, 344, 961-966.

Nisipeanu, P., \& Korczyn, A. D. (1993). Psychological stress as a risk factor for exacerbations in multiple sclerosis. Neurology, 43, 13111322.

Ockenfels, M. C., Porter, L., Smyth, J., Kirschbaum, C., Hellhammer, D. H., \& Stone, A. A. (1995). Effect of chronic stress associated with 
unemployment on salivary cortisol: Overall cortisol levels, diurnal rhythm, and acute stress reactivity. Psychosomatic Medicine, 57, 460467.

Paffenbarger, R. S., Blair, S. N., Lee, I., \& Hyde, R. T. (1993). Measurement of physical activity to assess health effects in a free-living population. Medicine and Science in Sports and Exercise, 25, 60-70.

Potter, P., \& Zautra, A. J. (1997). Stressful life events' effects on rheumatoid arthritis disease activity. Journal of Consulting and Clinical Psychology, 65, 319-323.

Pruessner, J. C., Hellhammer, D. H., \& Kirschbaum, C. (1999). Burnout, perceived stress, and cortisol responses to awakening. Psychosomatic Medicine, 61, 197-204.

Rabin, B. S. (1999). Stress, immune function, and health: The connection. New York: Wiley

Radloff, L. S. (1977). The CES-D scale: A self-report depression scale for research in the general population. Journal of Applied Psychological Measurement, 1, 385-401.

Rohleder, N., Schommer, N. C., Hellhammer, D., Engel, R., \& Kirschbaum, C. (2001). Sex differences in glucocorticoid sensitivity of proinflammatory cytokine production at baseline and after psychosocial stress. Psychosomatic Medicine, 63, 966-972.

Rozanski, A., Blumenthal, J. A., \& Kaplan, J. R. (1999). Impact of psychological factors on the pathogenesis of cardiovascular disease and implications for therapy. Circulation, 99, 2192-2217.

Scheinman, R. I., Cogswell, P. C., Lofquist, A. K., \& Baldwin, A. S. (1995, October 13). Role of transcriptional activation of $\mathrm{I} \kappa \mathrm{B} \alpha$ in mediation of immunosuppression by glucocorticoids. Science, 270, 283-285.

Sephton, S. E., Sapolsky, R. M., Kraemer, H. C., \& Spiegel, D. (2000). Diurnal cortisol rhythm as a predictor of breast cancer survival. Journal of the National Cancer Institute, 92, 994-1000.

Sheridan, J. F., \& Dobbs, C. M. (1994). Stress, viral pathogenesis, and immunity. In R. Glaser \& J. K. Kiecolt-Glaser (Eds.), Handbook of human stress and immunity (pp. 101-124). New York: Academic Press.

Sheridan, J. F., Dobbs, C. M., Jung, J., Chu, X., Konstantinos, A., Padgett, D. A., \& Glaser, R. (1998). Stress-induced neuroendocrine modulation of viral pathogenesis and immunity. Annals of the New York Academy of Sciences, 840, 803-808.

Smits, H. H., Grunberg, K., Derijk, R. H., Sterk, P. J., \& Hiemstra, P. S. (1998). Cytokine release and its modulation by dexamethasone in whole blood following exercise. Clinical \& Experimental Immunology, 111, 463-468.

Smyth, J. M., Ockenfels, M. C., Gorin, A. A., Catley, D., Porter, L. S., Kirschbaum, C., et al. (1997). Individual differences in the diurnal cycle of cortisol. Psychoneuroendocrinology, 22, 89-105.

Stark, J. L., Avitsur, R., Padgett, D. A., Campbell, K. A., Beck, F. M., \& Sheridan, J. F. (2001). Social stress induces glucocorticoid resistance in macrophages. American Journal of Physiology: Regulatory, Integrative and Comparative Physiology, 280, 1799-1805.

Sternberg, E. M., Hill, J. M., Chrousos, G. P., Kamilaris, T., Listwak, S. J.,
Gold, P. W., \& Wilder, R. L. (1989). Inflammatory mediator-induced hypothalamic-pituitary-adrenal axis activation is defective in streptococcal cell wall arthritis-susceptible Lewis rats. Proceedings of the National Academy of Sciences, USA, 86, 2374-2378.

Sternberg, E. M., Young, W. S., Bernardini, R., Calogero, A. E., Chrousos, G. P., Gold, P. W., \& Wilder, R. L. (1989). A central nervous system defect in biosynthesis of corticotropin-releasing hormone is associated with susceptibility to streptococcal cell wall-induced arthritis in Lewis rats. Proceedings of the National Academy of Sciences, USA, 86, 47714775.

Stone, A. A. (1992). Selected methodological concepts: Mediation and moderation, individual differences, aggregation strategies, and variability of replicates. In N. Schneiderman, P. McCabe, \& A. Baum (Eds.), Stress and disease processes: Perspectives in behavioral medicine (pp. 55-71). Hillsdale, NJ: Erlbaum.

Stone, A. A., Schwartz, J., Smyth, J., Kirschbaum, C., Cohen, S., Hellhammer, D., \& Grossman, S. (2001). Individual differences in the diurnal cycle of salivary free cortisol: A replication of flattened cycles for some individuals. Psychoneuroendocrinology, 26, 295-306.

Uchino, B. N., Cacioppo, J. T., \& Kiecolt-Glaser, J. K. (1996). The relationship between social support and physiological processes: A review with emphasis on underlying mechanisms and implications for health. Psychological Bulletin, 119, 488-531.

Usala, P. D., \& Hertzog, C. (1989). Measurement of affective states in adults: Evaluation of an adjective rating scale instrument. Research on Aging, 11, 403-426.

Weiner, H. (1992). Perturbing the organism: The biology of stressful experience. Chicago: University of Chicago Press.

Whitacre, C. C., Cummings, S. D., \& Griffin, A. C. (1995). The effects of stress on autoimmune disease. In R. Glaser \& J. K. Kiecolt-Glaser (Eds.), Handbook of human stress and immunity (pp. 77-100). New York: Academic Press.

Wilckens, T., \& DeRijk, R. (1997). Glucocorticoids and immune function: Unknown dimensions and new frontiers. Immunology Today, 18, 418 424.

Wright, R. J., Rodriquez, M. S., \& Cohen, S. (1998). Review of psychosocial stress and asthma: An integrated biopsychosocial approach. Tho$\operatorname{rax}, 53,1066-1074$.

Yehuda, R. (1998). Psychoneuroendocrinology of post-traumatic stress disorder. Psychiatric Clinics of North America, 21, 359-379.

Yehuda, R. (2000). Biology of post-traumatic stress disorder. Journal of Clinical Psychiatry, 61, 14-21.

Yehuda, R., Teicher, M. H., Trestman, R. L., Levengood, R. A., \& Siever, L. J. (1996). Cortisol regulation in posttraumatic stress disorder and major depression: A chronobiological analysis. Biological Psychiatry, 40, 79-88.

Zautra, A. J., Burleson, M. H., Matt, K. S., \& Roth, S. A. (1994), Interpersonal stress, depression, and disease activity in rheumatoid arthritis and osteoarthritis patients. Health Psychology, 13, 139-148. 